\title{
Simple Processes and the Pricing and Hedging of Cliquets
}

\author{
Dilip B. Madan \\ Robert H. Smith School of Business \\ University of Maryland \\ College Park, MD 20742 \\ email: dbm@rhsmith.umd.edu \\ Wim Schoutens \\ K.U.Leuven, Dept. of Mathematics \\ Celestijnenlaan 200B, \\ B-3001 Leuven, Belgium. \\ email: wim@schoutens.be
}

February 2, 2010

\begin{abstract}
For data on market prices on 246 cliquets we consider the task of pricing these exotic options using a relatively simple path space subsequently stressed to market implied and then predicted stress levels. An additive process transitioning from a Sato process to a Levy process is formulated and estimated on vanilla options. Ask prices constructed from predicted stress levels are observed to have an in sample correlation of $92 \%$ with market prices. Interestingly, it is observed that capped cash flows have negative stress levels while uncapped products have positive stress levels. We illustrate the effect of hedging cliquet liabilities using call options as hedging assets permiting a $10 \%$ reduction in ask prices.
\end{abstract}

We consider the possibility of pricing and hedging cliquets to prespecified levels of risk acceptability on simple path spaces. As a test case we take some 246 cliquets written on the SPX as an underlier with market valuations as at March 16 2009. The prospective hedging assets are vanilla options and hence we seek to consider models that reprice the vanilla surface. We wish to consider in the first instance the simplest models capable of fitting the surface of vanilla options. The simplest models are, like Brownian motion itself, processes of independent and identically distributed increments. This is the class of Lévy processes and it is well known that many members of this class will fit all vanilla option prices at a single maturity. Unfortunately, Lévy processes do not fit option prices across both strike and maturity and it is well known that for these processes skewness 
and excess kurtosis of the risk neutral density for the logarithm of the stock price decline respectively like the reciprocal of the square root of maturity and the reciprocal of maturity. The observed risk neutral entities are either constant or increasing.

The next simplest class of processes is the class of additive processes that still have independent increments but now they are inhomogeneous in time. Carr, Geman, Madan and Yor (2007) showed that this class of processes contained candidates they termed the Sato process that do fit options across all strikes and maturities up to two years with as few as four parameters synthesizing upto some 200 option prices. The marginal distributions of the Sato process are constructed by scaling the distribution at unit time and hence both skewness and excess kurtosis are constant across maturity.

We now have vanilla surfaces extending way past two years on many index underliers with maturities reaching up to 15 years. It is a little strange to continue the scaling operation for this long. For one, exponential moments will eventually fail to exist and on the other hand one expects that though skewness and excess kurtosis do not drop as fast as they do for Lévy processes but the impact of independent effects is to reduce them and it may be unrealistic to leave them constant for so long. These considerations led us to enquire into whether a Lévy process could fit risk neutral distributions for the logarithm of the stock price across maturities for maturities exceeding two years. We have over a hundred options now regularly quoted with such longer maturities. We were surprised to find that unlike the situation with the shorter maturities below a year or two, for the longer maturities many Lévy processes fit option surfaces across both strikes and maturities beyond two years. We were then confronted with the situation that the front end of the option surface was well described by a Sato process while the back end was consistent with a Lévy process.

This raised the question of constructing an additive process capable of transitioning from a Sato process for the lower maturities towards a Lévy process for the longer maturities. This is possible if one begins with a unit time distribution that is in the class $L^{1}$ in the hierarchy introduced by Urbanik $(1972,1973)$ and studied in detail by Sato (1980). Carr, Geman, Madan and Yor (2005) showed that the $C G M Y$ model has distributions in this class for $Y \geq .25$. This led us to the construction of what we termed the CGMYSatoLevy process that is presented in this paper.

We report on the calibration of this CGMYSatoLevy process to option prices on the $S \& P 500$ index as at March 16 2009. The calibrated parameters are then employed to generate a path space at a monthly reset with 10000 paths for 5 years and 60 monthly readings. Given the process additive one may simulate in large steps as the ratio of the marginal characteristic functions at two dates is itself a characteristic function and the forward return can be simulated using Fourier inversion and the inverse uniform method. We report on the CGMYSatoLevy price for all the 246 cliquets reported from for this date on the $S \& P 500$ index.

We also construct the target cash flows for each cliquet on this path space and ask what is the implied stress level using the stress function minmaxvar 
introduced in Cherny and Madan (2009). We observe that over $85 \%$ of the cliquets can be repriced using a stress level between \pm 0.75 . We then forecast the stress level using observable characteristics of the cliquet cash flows and show that we may explain $55 \%$ of the variation in the implied stress levels. We then use the predicted stress level to price the cliquet and obtain a correlation of $95 \%$ between the market price and our predicted price. Finally we investigate how far one may reduce the ask price by hedging a sample of cliquets to levels of acceptability determined by the implied stress levels.

The outline of the rest of the paper is as follows. Section 1 presents the evidence for Lévy processes calibrating longer maturity options across strikes and maturities. The CGMY SatoLevy process is developed in Section 2. Section 3 reports on the results of calibrating this process to March 162009 options on the $S \& P 500$ index. The resulting path space is evaluated in Section 4. Section 5 reports on the prices of the cliquets constructed on this path space. Section 6 . reports on the construction of the implied stress factors for the stress function minmaxvar and their properties. Section 7 takes up the task of explaining the stress factors. In Section 8 we use the predicted stress factors from section 7 to construct ask prices for the cliquet cash flows and report on their correlation with the market prices. Section 9 reports on the hedging of a sample of cliquets achieving price reductions of around 10\%. Section 10 concludes.

\section{$1 \quad$ Lévy Processes for the Back End}

We report in this section on the ability of Lévy processes to fit the option surface across strike and maturities exceeding two years. Two Lévy processes are considered, they are $C G M Y$ as presented in Carr, Geman, Madan and Yor (2002) and the spectrally negative form as studied in Eberlein and Madan (2009). The dynamics for the stock price $(S(t), t>0)$ in $C G M Y$ are specified as

$$
\begin{aligned}
d S & =(r-q) S d t+S_{-} \int_{-\infty}^{\infty}\left(e^{x}-1\right)\left(\mu_{C G M Y}(d x, d t)-k_{C G M Y}(x) d x d t\right) \\
k_{C G M Y}(x) & =C \frac{\exp \left(\frac{G-M}{2} x\right) \exp \left(-\frac{G+M}{2}|x|\right)}{|x|^{1+Y}} \mathbf{1}_{x \neq 0},
\end{aligned}
$$

where $\mu_{C G M Y}(d x, d t)$ is the counting measure for the jumps in the logarithm of the stock price, $r$ is the risk free interest rate, $q$ is the dividend yield and $k_{C G M Y}(x)$ is the arrival rate of a jump of size $x$.

It was observed in Eberlein and Madan (2009) that for back end options the densities tend to be consistent with Lévy processes with no positive jumps and we include a diffusion component to get a density with support on the whole 
line. This leads to the model $C G Y S N$ under which the stock dynamics are

$$
\begin{aligned}
d S & =(r-q) S d t+\sigma d W+S_{-} \int_{-\infty}^{0}\left(e^{x}-1\right)\left(\mu_{C G Y}(d x, d t)-k_{C G Y}(x) d x d t\right) \\
k_{C G Y}(x) & =C \frac{\exp (-G|x|)}{|x|^{1+Y}} \mathbf{1}_{x<0},
\end{aligned}
$$

where $(W(t), t>0)$ is a Brownian motion, $\mu_{C G Y}(d x, d t)$ is the counting measure

for the negative jumps in the logarithm of the stock price and $k_{C G Y}(x)$ is the jump arrival rate.

Both models were estimated on $S \& P 500$ index options with maturities exceeding 2 years as at March 16 2009. There were 147 options in this data set across 9 maturities ranging from 2.75 to just over 13 years. The estimation was conducted using the Carr and Madan (1999) methodology based on the characteristic function for the density of the logarithm. We report in Table 1 the results of the estimation.

$\begin{array}{lll}\text { TABLE } 1 \\ \text { Back End Lévy Process } \\ \text { Model } \\ \\ \text { CGMY } & C G Y S N \\ C, \sigma & 0.9121 & 0.0143 \\ G, C & 2.3218 & 0.9080 \\ M, G & 200.2568 & 2.3172 \\ Y & 0.00037 & 3.04 e-9 \\ \text { rmse } & 3.3806 & 3.3804 \\ \text { aae } & 2.9078 & 2.9069 \\ \text { ape } & 0.0181 & 0.0180\end{array}$

We observe that both models fit the data equally well. The value of $M$ being as alrge as it is suggests that a process with no positive jumps would be appropriate. Given the near zero value for $Y$ suggests that one could in fact work with the variance gamma model (Madan and Seneta (1990), Madan, Carr and Chang (1998)). We also present a graph of the fit of $C G Y S N$ to this option price data in Figure (1).

\section{The CGMYSatoLévy Process}

We recognize from Carr, Geman, Madan and Yor (2007) that the front end or maturities below a year or two are well explained by the additive Sato process, while as we have just observed the longer maturities are consistent with a Lévy process. In this section we report on the construction of an additive process that is capable of starting out as a Sato process and slowly transitioning into a Lévy process at the longer maturities. It is useful to briefly recall the construction of a Sato process. 


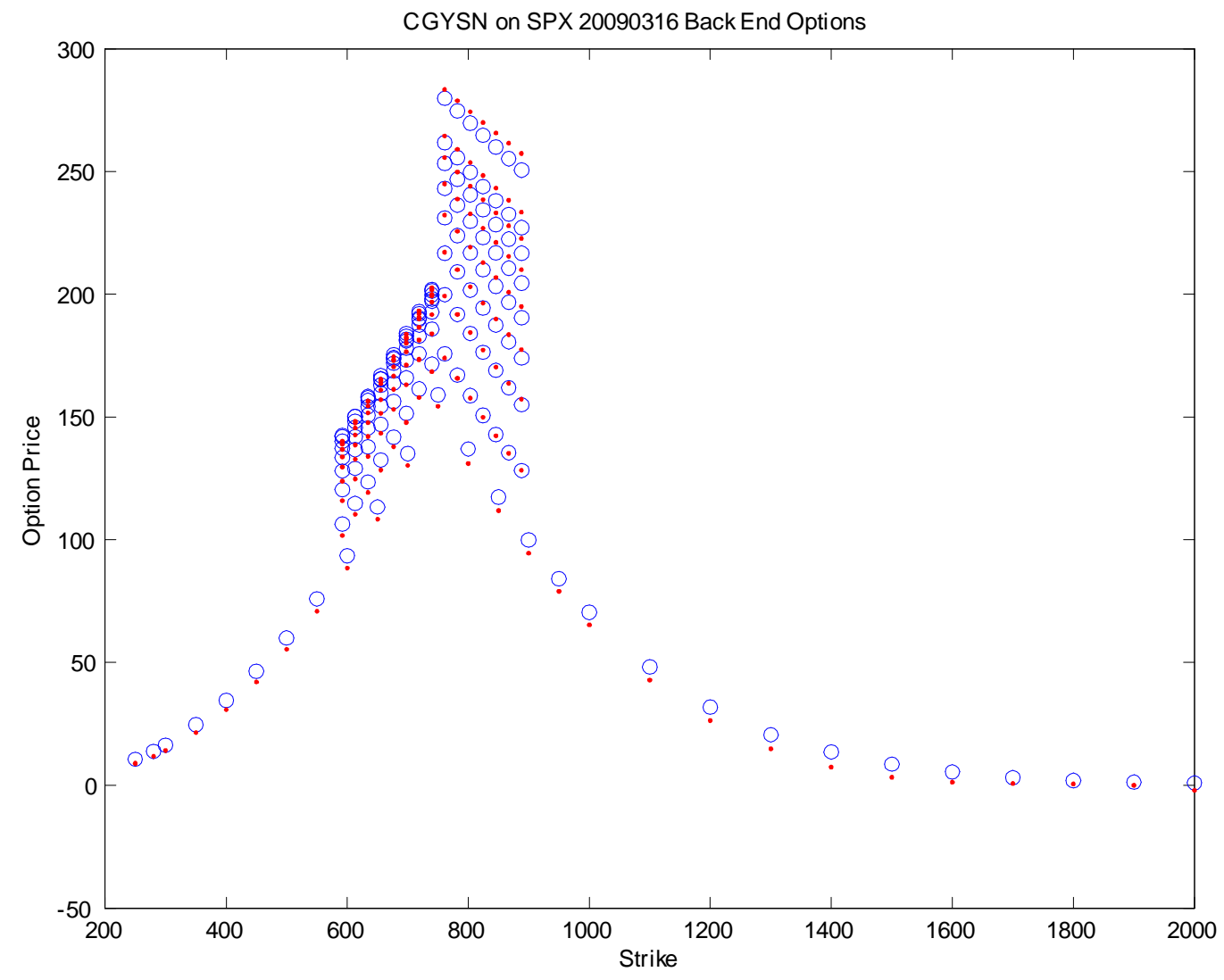

Figure 1: Option prices for all strikes with maturities exceeding two years are shown by circles. CGYSN model prices are represented by dots. 
For this one takes a self decomposable law $X$ at unit time and one then obtains the marginal laws at other time points $t$ by scaling using a scaling coefficient $\gamma$, defining

$$
X(t) \stackrel{(d)}{=} t^{\gamma} X
$$

Sato $(1991,1999)$ showed that if $X$ is self decomposable then there exists an additive process with independent and inhomogeneous increments with these marginals. The definition of self decomposability asserts that $X$ is self decomposable if and only if for every real number $c, 0<c<1$, there exists a random variable $X^{(c)}$ independent of $X$ such that

$$
X \stackrel{(d)}{=} c X+X^{(c)}
$$

Selfdecomposable laws are infinitely divisible with a special structure to their Lévy densities $k(x)$. In fact we must have that $h(x)=|x| k(x)$, is decreasing for positive $x$ and increasing for negative $x$. The symmetric variance gamma is a classic self decomposable law as $h(x)=\exp (-|x|)$. No jump diffusion can be selfdecomposable as such laws are necessarily of infinite activity with infinitely many jumps in any interval of time.

The stock price dynamics under a Sato process are given by

$$
\begin{aligned}
d S & =(r-q) S d t+S_{-} \int_{-\infty}^{\infty}\left(e^{x}-1\right)(\mu(d x, d t)-k(x, t) d x d t), \\
k(x, t) & =\frac{\operatorname{sign}(x) \gamma}{t^{1+\gamma}} h^{\prime}\left(\frac{x}{t^{\gamma}}\right) .
\end{aligned}
$$

The first step in the direction of constructing an additive process that can transition from Sato to Lévy was to follow the construction of long horizon returns from short horizon returns described in Eberlein and Madan (2009). Their interest in going to long horizon returns was to consider the decomposition (1) at unit time and to define the marginal at later time $t>1$ by running the Lévy process $(c X)$ to time $t$ and to scale the random variable $X^{(c)}$ at rate $\gamma$. This gave a distribution at time $t$ as

$$
X(t) \stackrel{(d)}{=}(c X)_{t}+t^{\gamma} X^{(c)} .
$$

The characteristic function of $X(t)$ is easily constructed in terms of the characteristic exponent $\psi_{X}(u)$ of $X$ as

$$
\begin{aligned}
E[\exp (i u X)] & =\exp \left(\psi_{X}(u)\right) \\
E[\exp (i u X(t))] & =\exp \left(t \psi_{X}(c u)+\psi_{X}\left(u t^{\gamma}\right)-\psi_{X}\left(c u t^{\gamma}\right)\right) .
\end{aligned}
$$

One may think of the coefficient $c$ as the proportion that is Lévy at time $t$. Furthermore one may estimate such marginal characteristic exponents and let the coefficient $c$ vary with the maturity to observe that in fact it rises towards unity as we increase maturity. In this way we get closer to a Lévy process for large $t$. For small $t$ we have $c$ near zero and one is scaling as in a Sato process. 
However, to ensure that all the marginals being constructed are consistent with an additive process we would like to have $X^{(c)}$ to be a selfdecomposable law so that the marginals obtained from scaling as in $t^{\gamma} X^{(c)}$, are associated with an additive process. For $X^{(c)}$ to be selfdecomposable it must be that $X$ itself was in the Urbanik class $L^{1}$. The class of self decomposable random variables satisfying condition (1) is the class $L^{0}$ and if (1) holds with $X^{(c)}$ itself in $L^{0}$ then $X$ is in $L^{1}$. We begin our construction with $X \in L^{1}$, and Carr, Geman, Madan and Yor (2005) showed that for the $C G M Y$ random variables, we must have $Y>.25$ to be in $L^{1}$. We now describe the detailed construction of the CGMY SatoLévy process.

The construction of a Sato Lévy process with a time varying instantaneous Lévy proportion $c(t)$ requires that we begin with a law at unit time that is in class $L^{1}$. For such a random variable $X$ we have that $X^{(c)}$ in (1) is itself self decomposable. In this case one may associate with $X^{(c)}$ a Sato process that matches the scaled marginals and we have that

$$
E\left[\exp \left(i u t^{\gamma} X^{(c)}\right)\right]=\exp \left(\int_{0}^{t} \int_{-\infty}^{\infty}\left(e^{i u x}-1\right) g(x, s) d x d s\right)
$$

for a Lévy system that may be identified in terms of the Lévy measure for $X^{(c)}$.

Consider now the process associated with a constant $c$ that runs $(c X)$ as a Lévy process and scales $X^{(c)}$. This process has characteristic exponent

$$
E\left[\exp \left(i u\left((c X)_{t}+t^{\gamma} X^{(c)}\right)\right)\right]=\exp \left(t \psi(c u)+\psi\left(u t^{\gamma}\right)-\psi\left(u c t^{\gamma}\right)\right)
$$

The Levy system associated with this characteristic exponent is

$$
\frac{1}{c} k\left(\frac{x}{c}\right) d x d t+g(x, t) d x d t
$$

where $k(x)$ is the Lévy measure of $X$ and $g$ is the Lévy system of the Sato process associated with $X^{(c)}$.

The Lévy measure of $X^{(c)}$ is easily derived from that of $X$ as its Lévy exponent is

$$
\begin{aligned}
E\left[\exp \left(i u X^{(c)}\right)\right] & =\exp (\psi(u)-\psi(c u)) \\
& =\exp \left(\int_{-\infty}^{\infty}\left(e^{i u x}-1\right) k(x) d x-\int_{-\infty}^{\infty}\left(e^{i u c x}-1\right) k(x) d x\right) \\
& =\exp \left(\int_{-\infty}^{\infty}\left(e^{i u x}-1\right)\left(k(x)-k\left(\frac{x}{c}\right) \frac{1}{c}\right) d x\right)
\end{aligned}
$$

It follows from the self decomposability of $X$ that

$$
k(x)-k\left(\frac{x}{c}\right) \frac{1}{c}
$$

is a Lévy density. 
In fact

$$
\operatorname{sign}\left(k(x)-k\left(\frac{x}{c}\right) \frac{1}{c}\right)=\operatorname{sign}\left(|x| k(x)-k\left(\frac{x}{c}\right) \frac{|x|}{c}\right)
$$

and the latter is positive by the self decomposability condition on Lévy densities.

We may now identify the Lévy system $g$ more specifically as

$$
\begin{aligned}
g(x, s) & =\frac{\operatorname{sign}(x) \gamma}{s^{1+\gamma}} h^{(c) \prime}\left(\frac{x}{s^{\gamma}}\right) \\
h^{(c)}(x) & =|x|\left(k(x)-k\left(\frac{x}{c}\right) \frac{1}{c}\right) \\
g(x, s) & =\frac{\operatorname{sign}(x) \gamma}{s^{1+\gamma}}\left(h^{\prime}\left(\frac{x}{s^{\gamma}}\right)-\frac{1}{c} h^{\prime}\left(\frac{x}{s^{\gamma} c}\right)\right)
\end{aligned}
$$

where $h$ is associated with the original Lévy process. The positivity of $g$ follows from the fact that $X^{(c)}$ is self decomposable.

We now construct a process that pieces together the processes with constant $c^{\prime} s$ but now we employ the coefficient $c_{i}$ in the interval $t_{i}, t_{i+1}$ for $t_{0}=0<t_{1}<$ $t_{2}<\cdots$. The Lévy exponent of this process in the interval $t_{0}<t<t_{1}$ is

$$
t \psi\left(c_{0} u\right)+\psi\left(u t^{\gamma}\right)-\psi\left(c_{0} u t^{\gamma}\right),
$$

and at $t_{1}$ we have the exponent

$$
t_{1} \psi\left(c_{0} u\right)+\psi\left(u t_{1}^{\gamma}\right)-\psi\left(c_{0} u t_{1}^{\gamma}\right) .
$$

In the interval $t_{1}<t<t_{2}$ we have the exponent

$$
\begin{aligned}
& t_{1} \psi\left(c_{0} u\right)+\psi\left(u t_{1}^{\gamma}\right)-\psi\left(c_{0} u t_{1}^{\gamma}\right)+ \\
& \left(t-t_{1}\right) \psi\left(c_{1} u\right)+\psi\left(u t^{\gamma}\right)-\psi\left(c_{1} u t^{\gamma}\right)-\psi\left(u t_{1}^{\gamma}\right)+\psi\left(c_{1} u t_{1}^{\gamma}\right)
\end{aligned}
$$

and at time $t_{2}$ we have

$$
\begin{aligned}
& t_{1} \psi\left(c_{0} u\right)+\psi\left(u t_{1}^{\gamma}\right)-\psi\left(c_{0} u t_{1}^{\gamma}\right)+ \\
& \left(t_{2}-t_{1}\right) \psi\left(c_{1} u\right)+\psi\left(u t_{2}^{\gamma}\right)-\psi\left(c_{1} u t_{2}^{\gamma}\right)-\psi\left(u t_{1}^{\gamma}\right)+\psi\left(c_{1} u t_{1}^{\gamma}\right) .
\end{aligned}
$$

In general at time $t_{k}$ we have the exponent

$$
\sum_{j=1}^{k}\left(t_{j}-t_{j-1}\right) \psi\left(c_{j-1} u\right)+\psi\left(u t_{j}^{\gamma}\right)-\psi\left(c_{j-1} u t_{j}^{\gamma}\right)-\psi\left(u t_{j-1}^{\gamma}\right)+\psi\left(c_{j-1} u t_{j-1}^{\gamma}\right)
$$

and for the general case we may write

$$
\begin{aligned}
& \sum_{j=1}^{n}\left(s_{j}-s_{j-1}\right) \psi\left(c_{j-1} u\right)+\psi\left(u s_{j}^{\gamma}\right)-\psi\left(c_{j-1} u s_{j}^{\gamma}\right)-\psi\left(u s_{j-1}^{\gamma}\right)+\psi\left(c_{j-1} u s_{j-1}^{\gamma}\right) \\
s_{j}= & \left(t \wedge t_{j}\right) .
\end{aligned}
$$


The Lévy system in the interval $t_{i}<t<t_{i+1}$ is

$$
\frac{1}{c_{i}} k\left(\frac{x}{c_{i}}\right) d x d s+\frac{h^{\prime}\left(\frac{x}{t^{\gamma}}\right) \gamma}{t^{1+\gamma}}-\frac{h^{\prime}\left(\frac{x}{t^{\gamma} c_{i}}\right) \gamma}{t^{1+\gamma} c_{i}} d x d t .
$$

We may also let $c$ depend continuously on time to get the Lévy system

$$
\frac{1}{c(t)} k\left(\frac{x}{c(t)}\right) d x d t+\frac{h^{\prime}\left(\frac{x}{t^{\gamma}}\right) \gamma}{t^{1+\gamma}}-\frac{h^{\prime}\left(\frac{x}{t^{\gamma} c(t)}\right) \gamma}{t^{1+\gamma} c(t)} d x d t
$$

The associated Lévy exponent is

$$
\left.\int_{0}^{t} \psi(c(s) u) d s+\int_{0}^{t} \psi^{\prime}\left(u s^{\gamma}\right) \gamma u s^{\gamma-1}-\psi^{\prime}\left(c(s) u s^{\gamma}\right) \gamma u c(s) s^{\gamma-1}\right) d s .
$$

One may independently verify that

$$
\begin{aligned}
& \int_{-\infty}^{\infty}\left(e^{i u x}-1\right) \frac{h^{\prime}\left(\frac{x}{s^{\gamma}}\right) \gamma}{s^{1+\gamma}}-\frac{h^{\prime}\left(\frac{x}{s^{\gamma} c(s)}\right) \gamma}{s^{1+\gamma} c(s)} d x \\
= & \psi^{\prime}\left(u s^{\gamma}\right) \gamma u s^{\gamma-1}-\psi^{\prime}\left(c(s) u s^{\gamma}\right) \gamma u c(s) s^{\gamma-1} .
\end{aligned}
$$

We formulate a smooth transition piecewise constant model for $c(t)$ by

$$
c\left(t_{i}\right)=c_{i}
$$

for $t$ intermediate between two points we take $t_{i}<t<t_{i+1}$. We may take the intervals $t_{i+1}-t_{i}$ to be small and this will give us many parameters $c_{i}$ to be estimated, however we may estimate at a few points but interpolate between these values at other points. In this way we avoid big jumps in the levels of $c_{i}$.

\section{Calibration for March 162009 on S\&P 500 Index}

We report on the results of estimation on data for options on the $S \& P 500$ index as at 20090316. Here we employed all options with maturities ranging from one month to just over 13 years. There were 228 out of the money options used in 
the calibration. The results of the estimation were as follows.

$$
\begin{aligned}
C & =0.1294 \\
G & =0.5553 \\
M & =140.5080 \\
Y & =0.9696 \\
\gamma & =0.49199 \\
c_{1} & =0 ; t_{1}=0.03 \\
c_{2} & =0 ; t_{2}=0.25 \\
c_{3} & =0 ; t_{3}=0.50 \\
c_{4} & =0 ; t_{4}=1.0 \\
c_{5} & =0.5220 ; t_{5}=2.0 \\
c_{6} & =0.6994 ; t_{6}=5.0 \\
c_{7} & =0.6731 ; t_{7}=7.0 \\
c_{8} & =0.7525 ; t_{8}=15.0 \\
R M S E & =2.2058 \\
A A E & =1.9232 \\
A P E & =0.0157 \\
M O P & =228 \\
N F N E V A L S & =4872
\end{aligned}
$$

We present a graph of the fit to option prices in Figure (2).

We observe that the Lévy proportion rises after one year to 0.52 at two years and 0.75 at 15 years. Below a year the calibration favors a strict Sato process and we observe a calibrated transition from Sato to Lévy as was anticipated by the results of the first section.

\section{Generating Path Spaces of Additive Processes}

Given that we have an additive process one may easily construct a forward characteristic function for log price retruns by taking the ratio of the marginal characteristic functions at two successive maturities. This function may then be used to generate the forward return cumulative distribution function for a monthly horizon and using the inverse uniform method one generates path spaces consistent with this process. We generated a matrix of 60 rows and 10000 columns for a five year monthly time step giving us 10000 draws on 60 inhomogeneous monthly returns.

We first report on the pricing of vanilla options on this path space for a set 


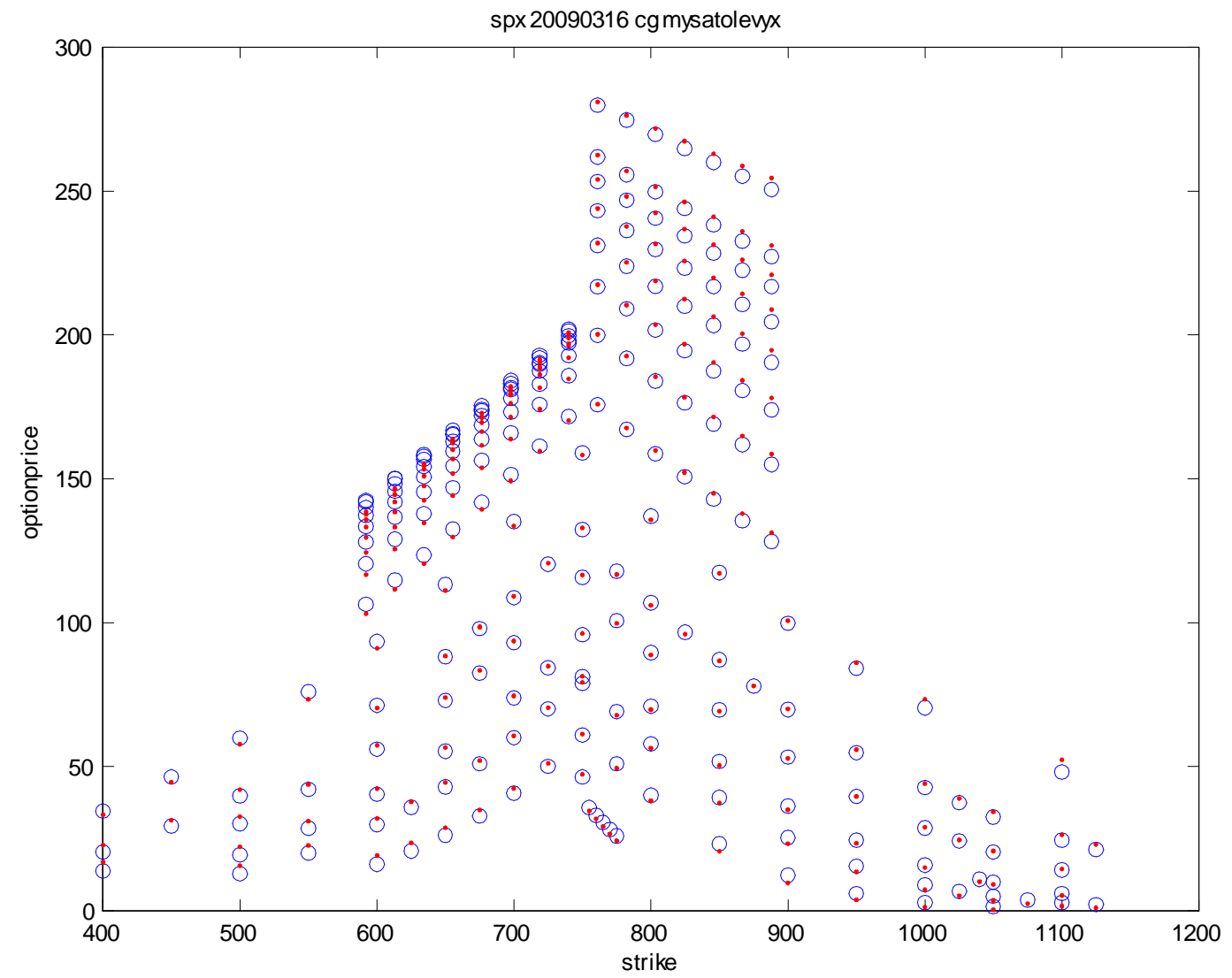

Figure 2: Fit of cgmysatolevyx to SPX options on 20090316. 
of strikes at three year ends of one two and five years.

\begin{tabular}{lll}
\multicolumn{2}{l}{ One Year } \\
Strike & Model & Simulation \\
80 & 6.6226 & 6.5424 \\
88 & 9.1485 & 9.0731 \\
96 & 12.3199 & 12.2297 \\
104 & 12.1926 & 12.0798 \\
112 & 8.7896 & 8.6578 \\
120 & 6.0949 & 5.9462
\end{tabular}

Two Year

$\begin{array}{lll}\text { Strike } & \text { Model } & \text { Simulation } \\ 80 & 10.3858 & 10.2647 \\ 88 & 13.4860 & 13.3443 \\ 96 & 17.1076 & 16.9556 \\ 104 & 17.2552 & 17.0926 \\ 112 & 13.9193 & 13.7334 \\ 120 & 11.0777 & 10.8532\end{array}$

Five Year

$\begin{array}{lll}\text { Strike } & \text { Model } & \text { Simulation } \\ 80 & 18.6069 & 18.3562 \\ 88 & 22.6525 & 22.3869 \\ 96 & 27.0518 & 26.7686 \\ 104 & 27.7831 & 27.4997 \\ 112 & 24.8238 & 24.5412 \\ 120 & 22.1514 & 21.8539\end{array}$

\section{Pricing Cliquets on the Path Space}

For this date we have market quotes on 246 cliquets with maturities ranging from one to five years and reset periods being, annual, monthly, quarterly or semi-annual. There are 90 options that are equally divided between rolling calls and rolling puts struck at or out of the money employing three strikes on either side. There are in addition 36 options that are just locally capped and 48 that are just locally floored and 72 that are both locally floored and locally capped.

The product definitions are

$$
1 m R P, 3 m R C
$$

for a one month rolling put or a three month rolling call.

In addition we have

$$
1 m 4 L C, 3 m 4 L C 3 L F, 6 m 6 L F
$$


for a one month locally capped cliquet with a $4 \%$ cap, a 3 month locally capped at $4 \%$ and locally floored cliquet at $-3 \%$ and a 6 month locally floored cliquet at $6 \%$ down.

We employed our path space to price all 246 options, and a sample of results are presented in the next section.

\section{Constructing Implied Stress Levels}

We employ the theory of indices of acceptability of random cash flows introduced in Cherny and Madan (2009) to compute the implied stress level at which each cliquet is priced. This theory basically operationalizes the concept of acceptable risks introduced in Artzner, Delbaen, Eber and Heath (1998) and studied further in Carr, Geman and Madan (2001), Jaschke and Küchler (2001) from the perspective of generalizing the absence of arbitrage. Cherny and Madan (2009) provide examples of effectively computing such sets of acceptable risks using the theory of concave distortions also studied in Föllmer and Schied (2004).

An application requires the choice of a parameterized family of concave distribution functions from the unit interval to the unit interval where the parameter say $\gamma$, raises the degree of concavity as one increases the value of $\gamma$ with the functions approaching unity pointwise for all arguments above zero. The distortion recommended in Cherny and Madan (2009) is the minmaxvar function defined by

$$
\Psi^{\gamma}(u)=1-\left(1-u^{\frac{1}{1+\gamma}}\right)^{1+\gamma} 0 \leq u \leq 1 .
$$

A random cash flow $C$ with distribution function $F(c)$ is acceptable at level $\gamma$ provided

$$
\int_{-\infty}^{\infty} c d \Psi^{\gamma}(F(c)) \geq 0
$$

If a cliquet is sold for an up front ask price that makes the residual cash flow of the price less the promised cash flow acceptable at level $\gamma$, then it is shown in Madan (2009) that the resulting ask price, $a$, is given by

$$
a=-\int_{-\infty}^{\infty} x d \Psi^{\gamma}\left(F_{(-X)}(x)\right)
$$

where $F_{(-X)}(x)$ is the distribution function of the cliquet liability.

For each cliquet we compute the implied stress level by solving for $\gamma$ such that

$$
\text { Market price }=-\int_{-\infty}^{\infty} x d \Psi^{\gamma}\left(F_{(-X)}(x)\right) .
$$

We may not always be able to find the implied stress level and in this study we matched all but 5 of the 246 prices. We note further that Cherny and Madan (2009) worked with the physical measure as the base measure and treated all cash flows with a negative expectation as unacceptable with the result that all stress levels are positive. Our path space here is a risk neutral path space that 
is already stressed and we permit negative values of $\gamma$ in matching the market price. One is then just unstressing the already stressed initial path space.

We present in Table 2 a sample of cliquet prices and the associated stress levels.

Table 2

Market Prices, Model Prices and Stress Levels

\begin{tabular}{|c|c|c|c|c|c|}
\hline Maturity & Strike & $\begin{array}{l}\text { Cliquet } \\
\text { Product }\end{array}$ & $\begin{array}{l}\text { Market } \\
\text { price }\end{array}$ & $\begin{array}{l}\text { cgmysatolevy } \\
\text { model price }\end{array}$ & $\begin{array}{l}\text { minmaxvar } \\
\text { stress level }\end{array}$ \\
\hline 12 & 94 & $1 \mathrm{mRP}$ & 24.08 & 19.94 & .1104 \\
\hline 24 & 90 & $3 \mathrm{mRP}$ & 26.20 & 21.89 & 1003 \\
\hline 60 & 100 & $6 \mathrm{mRP}$ & 83.42 & 67.76 & 2099 \\
\hline 12 & 107 & $6 \mathrm{mRC}$ & 12.45 & 10.66 & .0972 \\
\hline 24 & 105 & $3 \mathrm{mRC}$ & 33.14 & 21.82 & .5662 \\
\hline 60 & 100 & $1 \mathrm{mRC}$ & 206.63 & 138.33 & 4.0986 \\
\hline 12 & & $1 \mathrm{~m} 4 \mathrm{LC}$ & 2.88 & 5.44 & -.2164 \\
\hline 24 & & $3 \mathrm{~m} 6 \mathrm{LC}$ & 3.91 & 5.83 & -.1406 \\
\hline 60 & & 1m4LC3LF & 40.84 & 58.17 & -.4238 \\
\hline 12 & & 6m8LC6LF & 4.98 & 5.66 & -.0722 \\
\hline 60 & & 1m3LF & 144.66 & 96.08 & 1.5103 \\
\hline 24 & & $6 \mathrm{~m} 6 \mathrm{LF}$ & 28.57 & 24.77 & .1102 \\
\hline
\end{tabular}

\section{Predicting Stress Factors}

We next considered the task of predicting the stress factors from product characteristics that could be observed from the product definition and the model prices generated by our path space. One may conjecture that stress factors may posses a term structure related to their maturity. In addition we observed interestingly that all products with a capped cash flow had a negative stress factor and all products with a positive stress factor had uncapped cash flows. Hence we used a dummy variable that was unity if the product had a capped cash flow. In addition we used the model price and its square. We therefore had 8 explanatory variables in a regression of stress levels on 5 maturity dummies, one dummy for capped cash flows along with the model price and its square. 
The results of the regression are reported in Table 3.

\begin{tabular}{lll}
\multicolumn{2}{l}{$\begin{array}{l}\text { Table } 3 \\
\text { Stress Level Regression }\end{array}$} & \\
Variable & Coefficient & t-value \\
one year & 0.5655 & 5.95 \\
two year & 0.7306 & 6.64 \\
three year & 0.9806 & 6.41 \\
four year & 0.7863 & 4.83 \\
five year & 0.6891 & 5.25 \\
capped & -0.6810 & -7.71 \\
model price & -2.0233 & -5.18 \\
squared model price & 2.6104 & 8.59 \\
$\mathrm{R}^{2}$ & $55.38 \%$ &
\end{tabular}

The underlying stress levels are significantly related to a variety of product characteristics. There is a clear term structure with stress levels rising with maturity up to three years and fallin thereafter. Stress levels are reduced for capped cash flows and we have observed that they are in fact negative in this case. Low valued products have a lower stress level that rises quadratically with the model price.

\section{Using Predicted Stress Level to Price Cliquets}

In this section we report on how ask prices computed using equation (2) at stress levels predicted from our regression compare with the reported market prices. We present the result of regressing the market price on the ask price, ap, computed at the predicted stress level.

$$
\text { market price }=.0566+1.3345 * a p
$$

The figures in brackets are $t$-values for the null hypothesis of zero and unity respectively. The $R^{2}$ was $91.55 \%$. The hypothesis that market prices come from these stress adjustments is formally rejected but the methodology pro-

duces results that are reasonable, given the expected variation in market prices themselves.

We present additionally in Figure (3) a graph of the market prices against the ask price generated from predicted stress levels.

\section{Hedging a sample of cliquets to acceptable risk levels}

Given that stress levels are positive for uncapped cash flows, we take two such cash flows with a relatively high stress level and investigate how one may reduce 


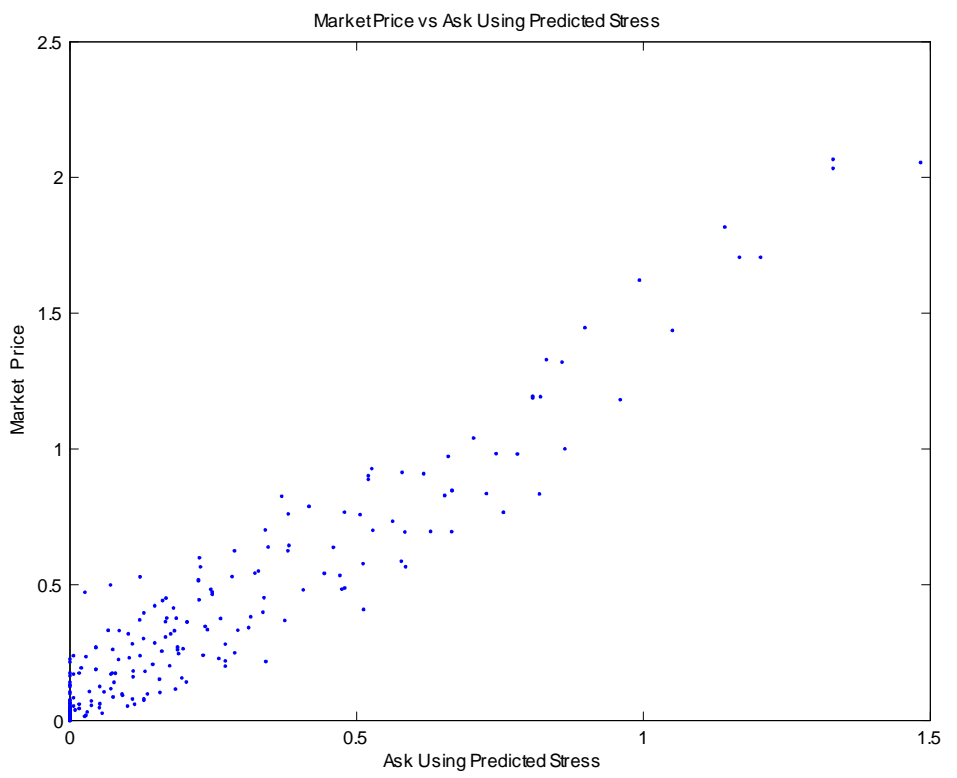

Figure 3: Market Price vs Ask price from predicted stress levels on cgmysatolevy additive pathspace

the cost by constructing a hedge on our path space. We take two examples. The first is a one month five year at the money rolling call with a market price of 206.63 and an implied stress level of 4.0986. The second is a five year, one month cliquet locally floored at $3 \%$ with a market price of 144.66 and an implied stress level of 1.5103 .

Since the risk is the uncapped cash flow on the upside, we take for hedging assets call options at 9 strikes ranging from 80 to 120 in five dollar intervals. We take positions in these 9 calls at all of 20 maturities given by the 20 quarters over the five year period. There are therefore 180 hedging call options and we use our pathspace to construct a matrix of dimension 180 by 10000 for the cash flows to these 180 calls on each of 10000 paths. The price of the option is subtracted and the hedge cash flows are therefore zero cost cash flows. Equivalently the purchase of options is financed with payment at the end of the five year period. We denote this matrix by $H$.

For each of our two products cash flows we have constructed the vector of the target cash flow that we denote by $Y$. For a prospective hedge position of $x$ given by a vector of dimension 180 , the final cash flow to the cliquet seller is

$$
C=x^{\prime} H-Y \text {. }
$$

The objective function to be minimized is the ask price given by equation (2) with $C$ in the role of $X$, and the stress level used is the implied stress level. 


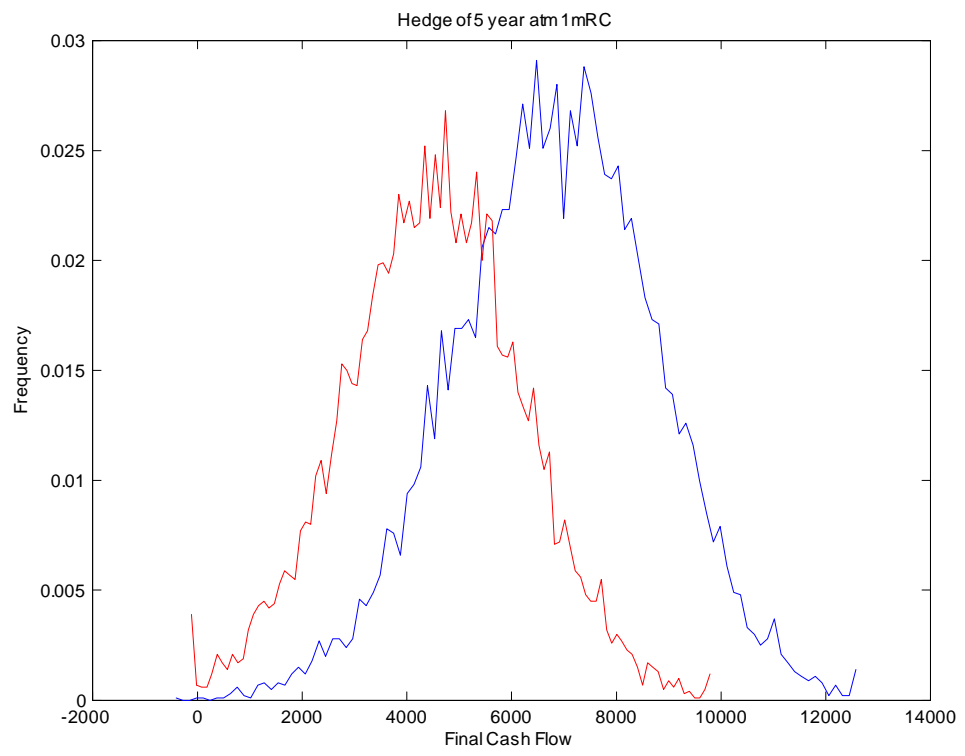

Figure 4: Histograms of hedged and unhedged cash flows for 5 year at the money rolling call.

For a zero hedge the objective function then evaluates to the market prices of 206.63 and 144.66 for our two products.

In each case we minimized the objective function over the 180 dimensional search for the minimal ask price to reduce the respective ask prices to 183.48 and 130.10 , a reduction of around $10 \%$ in the price. We present the graphs of the historgams for final cash flows inclusive of price, payment of liability and hedge earnings for the two products on our path space. For the first product the price from the perspective of our path space was close to a sample space arbitrage, with some but few negative outcomes. The hedge lowers the price, reduces the volatility and lowers the price, maintaining the comparably high level of acceptability. For the second product, the loss region, volatility and price are all reduced by the hedge, again maintaining the level of acceptability at the now lower, but still substantial implied stress level.

\section{Conclusion}

We consider the task of pricing cliquets using a relatively simple path space generated from an additive process that permits an easy large step simulation synchronized to the cliquet reset period. For a test case we use data on 246 cliquet prices on the $S \& P 500$ index as at March 16 2009. Models are calibrated to the vanilla option surface for the $S \& P 500$ index on this date. 


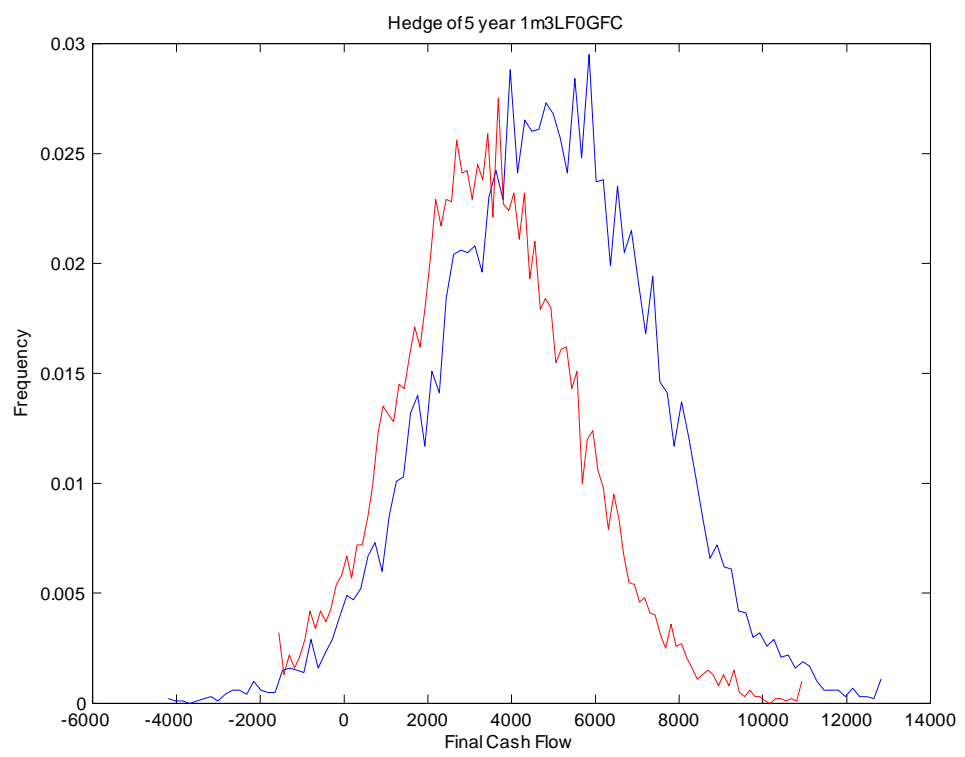

Figure 5: Histogram of hedged and unhedged cash flows for 5 year one month 3 percent locally floored cliquet

It is first observed that though Lévy processes are known not to fit the option surface at maturities below a year they perform quite well for maturities above two years. For options under a year the Sato process provides a good calibration as noted in Carr, Geman, Madan and Yor (2007). The Sato process is an additive process and the question then arises as to how one may construct an additive process that transitions from Sato towards a Lévy process as the maturity is increased. Such a process is constructed in this paper starting from a law in the Urbanik $(1972,1973)$ class $L^{1}$. We base our construction on the $C G M Y$ with $Y \geq 0.25$ to ensure that we are in $L^{1}$. This model is termed CGMY SatoLevy.

We calibrate this model to the vanilla options, generate path spaces from this process, and price all 246 cliquets. We then go on to consider the construction of implied stress levels for the minmaxvar stress function introduced in Cherny and Madan (2009) and employed in Madan (2009) to generate ask prices and hedges for basket options. Here we generate the implied stress levels consistent with the market prices.

We then predict these stress levels, observing interestingly, that capped cash flows have negative stress levels while uncapped cash flows have positive stress levels. Ask prices constructed from predicted stress levels are observed to have an in sample correlation of $92 \%$ with market prices. The overall structure and methodology is therefore potentially promising for a more detailed out of sample study of cliquet pricing. 
Finally we illustrate the effect of hedging cliquet liabilities on our path space to minmaxvar levels of acceptability as calibrated by implied stress levels. The use of call options to limit uncapped liabilities as hedging assets permits a $10 \%$ reduction in price while maintaining acceptability levels.

\section{References}

[1] Artzner, P., Delbaen, F., Eber, J., Heath, D. (1998), "Definition of coherent measures of risk," Mathematical Finance 9, 203-228.

[2] Carr, P., H. Geman, H., and D. B. Madan (2001), "Pricing and Hedging in Incomplete Markets," Journal of Financial Economics 62, 131-167.

[3] Carr, P. and D. B. Madan (1999), "Option Pricing Using the Fast Fourier Transform," Journal of Computational Finance, 2, 61-73.

[4] Cherny, A. and D. B. Madan (2009), "New Measures for Performance Evaluation," Review of Financial Studies, 22, 2571-2606.

[5] Carr, P., H. Geman, D. Madan and M. Yor (2002), "The Fine Structure of Structure of Asset Returns: An Empirical Investigation," Journal of Business, 75, 305-332.

[6] Carr, P., H. Geman, D. Madan and M. Yor (2007), "Self Decomposability and Option Pricing," Mathematical Finance, 17, 31-57.

[7] Carr, P., H. Geman, D. Madan and M. Yor (2005), "Pricing Options on Realized Variance," Finance and Stochastics, 9, 453-475.

[8] Eberlein, E. and D. B. Madan (2009), "Short Sale Restrictions, Rally Fears and Option Pricing," Applied Mathematical Finance, forthcoming.

[9] Föllmer, H. and A. Schied (2004), Stochastic Finance: An Introduction in Discrete Time, Walter de Gruyter, Berlin.

[10] Jaschke, S. and U. Küchler (2001), "Coherent Risk Measures and Good Deal Bounds," Finance and Stochastics, 5, 181-200.

[11] Madan, D. B. , P. Carr and E. Chang (1998), "The Variance Gamma Process and Option Pricing," European Finance Review, 2,79-105.

[12] Madan, D. B. and E. Seneta (1990) "The V.G. Model for Share Market Returns," Journal of Business, 63,4,511-52.

[13] Sato, K. (1980), "Class L of multivariate distributions and its subclasses," Journal of Multivariate Analysis, 10, 207-232.

[14] Sato, K. (1991), "Self similar processes with independent increments," Probability Theory and Related Fields, 89, 285-300. 
[15] Sato, K. (1999), Lévy processes and Infinitely Divisible Distributions, Cambridge University Press, Cambridge.

[16] Urbanik, K. (1972), "Slowly varying sequences of random variables," Bull. Polon. Sci. Sér. Sci. Astronom. Phys. 20, 679-682.

[17] Urbanik, K. (1973), "Limit laws for sequences of normed sums satisfying some stability conditions," Multivariate Analysis-III (P.R.Krishnaiah, ed.) Academic Press, New York, 225-237. 